\title{
Buchbesprechung
}

Friedrich Curtius, Individum und Krankheit. Grundzüge einer Individualpathologie. VII, 467 S. m. 58 z. T. farb. Abb. Springer-Verlag, Berlin-GöttingenHeidelberg 1959 Ln. DM 88,-

Die diagnostische Beräcksichtigung der individuellen Besondel heiten bei den Krankheitserscheinungen des Mensehen, diese alte Forderung gewinnt fraglos in letzter Zeit immer gröbere Bedeutung und Beachtung, hat aber bisher noch keine ernsthafte und gründliche Bearbeitung gefunden. Gegenüber einer vielfach vielleicht überstarken schematisierenden Pathophysiologie versucht das vorliegende Buch zur Pathologie des Individuums rorzudringen und damit das Verständnis für die biologische Konstellation des Einzelkranken zu wecken. Ein überaus bemerkenswertes und verdienstvolles Buch, das auch für den Zahnarzt und den Kieferorthopäden im Hinblick auf seine ständige Auseinandersetzung mit dem ...indiriduellen biologisehen Faktor" "im Mund- und Kieferbereich großes Interesse besitzen dïifte.

Der Verfasser bietet in seinem Buch eine reiche Sammlung klinischen Beobachtungsgutes, oft ergänzt durch die Befunde am Sektionstisch, und zeigt so an vielen konkreten Beispielen die durch keine Laboratoriumstechnik erfaßbare individuelle Varjabilität und Komplexität mancher Krankheiten auf.

Der nicht immer leichte Stoff ist in fün Hauptabschnitte gegliedert, deren Überschriften ..Indiridualität und Krankheitsentsteinung" (mit den Kapiteln: die Krankheitsveruisachung, der prämorbide Zustand, die individuelle Reaktionsweise, die Organdisposition). ..Individualität und Krankheitsgestaltung“ (mit einem Abschnitt „Krankheit und Persönlichkeit"), ..Individualität und Krankheitsbeurteilung" sowie ,Individualität u. Therapie" bereits den außerordentlich reichhaltigen Inhalt dieses Werkes erkennen lassen. Aus der ausführlichen Besprechung einer umfangreichen Kasuistik ergibt sich eine ungeahnte Fülle neuer Erkenntnisse und es wächst schließlich eine grundsätzliche Präzisiesung der gestellten Problematik und eine zusammenfassende thleitung sich ergebender Zusammenhänge und Folgerungen.

Das Curtiussche Buch füllt zweifellos eine Lücke aus. Dank seiner vorzüglichen textlichen Darstellung und klaren systematischen Gliederung kann sein aufmerksames Studium dem Kliniker und praktischen Arzt und nicht zuletzt auch dem Zahnarzt, der sich über die (irenzen seines engeren Fachgebietes orientieren will, nur bestens empfchlen werden. Die Alsstattung des Buches ist vorzüglich; das Literaturverzeichnis vermittelt wohl einen lïckenlosen Überblick des einschlägigen Schrifttums.

Alles in allem eine bemerkenswerte. auf hohem wissenschaftlichem Niveau stehende, kraftrolle literarische Leistung!

G. Korkhaus (Bom)

\section{Berichtigung zu Bd. 22, Heft 4}

Auf Seite 483 muß es richtig heißen

Kieferorthopädische Hilfe bei Blasinstrumentalisten

Von Dr. Gerd A. Schneider, Erfurt

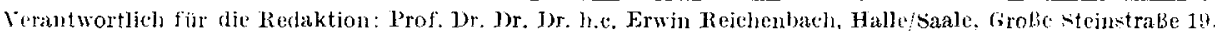

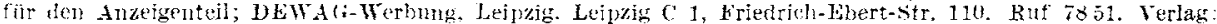

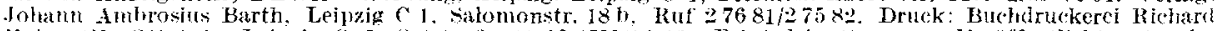

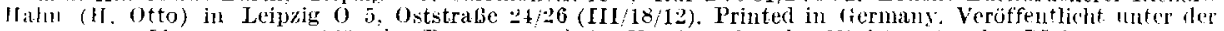
Lizenzummer 1391 des Pressatutes heim Forsitzenden des Minjsterrates der DUli 